\title{
Learning as program induction
}

\author{
Neil R. Bramley ${ }^{1}$, Eric Schulz ${ }^{2}$, Fei Xu ${ }^{3} \&$ Joshua B. Tenenbaum ${ }^{4}$ \\ ${ }^{1}$ Department of Psychology and Center for Data Science, New York University \\ ${ }^{2}$ Department of Psychology, Harvard University \\ ${ }^{3}$ Department of Psychology, University of California, Berkeley \\ ${ }^{4}$ Department of Brain and Cognitive Sciences, Massachusetts Institute of Technology
}

Keywords: program induction; hypothesis generation; discovery; concept learning; generalization; problem solving

\section{Introduction}

This workshop will cover new work that casts human learning as program induction - i.e. learning of programs from data.

The notion that the mind approximates rational (Bayesian) inference has had a strong influence on thinking in psychology since the 1950s. In constrained scenarios, typical of psychology experiments, people often behave in ways that approximate the dictates of probability theory. However, natural learning contexts are typically much more open-ended there are often no clear limits on what is possible, and initial proposals often prove inadequate. This means that coming up with the right hypotheses and theories in the first place is often much harder than ruling among them. How do people, and how can machines, expand their hypothesis spaces to generate wholly new ideas, plans and solutions? Recent work has begun to shed light on this problem via the idea that many aspects of learning can be better understood through the mathematics of program induction (Chater \& Oaksford, 2013; Lake, Salakhutdinov, \& Tenenbaum, 2015).

People are demonstrably able to compose hypotheses from parts (Goodman, Tenenbaum, Feldman, \& Griffiths, 2008; Piantadosi, Tenenbaum, \& Goodman, 2016; Schulz, Tenenbaum, Duvenaud, Speekenbrink, \& Gershman, 2017) and incrementally grow and adapt their models of the world (Bramley, Dayan, Griffiths, \& Lagnado, 2017). A number of recent studies has formalized these abilities as program induction, using algorithms that mix stochastic recombination of primitives with memoization and compression to explain data (Dechter, Malmaud, Adams, \& Tenenbaum, 2013; Ellis, Dechter, \& Tenenbaum, 2015; Romano, Salles, Amalric, Dehaene, Sigman, \& Figueria, 2017), ask informative questions (Rothe, Lake, \& Gureckis, 2017), and support one- and few-shot-inferences (Lake et al., 2015). Program induction is also proving to be an important notion for understanding development and learning through play (Sim \& Xu, 2017) and the formation of geometric understanding about the physical world (Amalric, Wang, Pica, Figueira, Sigman, \& Dehaene, 2017).

\section{Goal and scope}

The aim of our workshop is thus to bring together scientists who have a joint interest in how intelligent systems (humans or machines) can learn rich representations and action plans (expressable as programs) through observing and interacting with the world. We have invited leading researchers from cognitive science, linguistics, and artificial intelligence interested in the computational foundations of reasoning.

In particular, our goal is to bring together researchers working on broad range of topics related to program induction so as to facilitate discussion and help this nascent but exciting research area mature into a powerful and general theory of learning. Key topics of discussion will include (but are not limited to):

- How should we formalize and test different theories of program induction in human cognition?

- What is a good framework and what are good domains in which to benchmark progress in program induction in machine learning, and as a general theory of learning?

- What can diverse fields such as linguistics, symbolic systems, computational logic, contribute to one another's individual projects and the overarching theory?

- What are the necessary ingredients for a compositional theory of cognition? I.e., what primitives do people have? What modes of composition? Methods of compression? Learning-to-learn ability?

- What experiments will help separate the unique predictions of program induction from those of traditional accounts of compositionality and conceptual change (cf. Bruner, Goodnow, \& Austin, 1956)?

\section{Target audience}

This workshop dovetails neatly with this year's focus on "change, learning, growth, and adaptation". These key elements of cognition are precisely those that have resisted Bayesian accounts, and those which learning as program induction theories purport to explain. Our target audience is almost as broad as the conference as a whole - we expect this workshop to be of interest to psychologists, linguists, philosophers and machine learning researchers alike. Moreover, we feel that the interdisciplinary nature of our workshop will facilitate interactions between the diverse strands of research presented at CogSci that might otherwise remain bunkered in parallel sessions. Workshop page can be found at: https://programinduction.github.io

\section{Organizers and presenters}

The following presenters have confirmed their attendance:

Neil R. Bramley (Organizer) is a Postdoc at NYU working in Todd Gureckis' Computation and Cognition lab and the 
Center for Data Science. Neil's work explores how people actively learn, represent, and reason about the physical world. Eric Schulz (Organizer) is a Data Science Postdoctoral Fellow at Harvard University working in Samuel J. Gershman's Computational Cognitive Neuroscience lab. Eric studies generalization as function learning with a particular focus on compositionality and reinforcement learning.

Fei Xu (Organizer) is a Professor of Developmental Psychology at UC Berkeley. Fei's lab focuses on cognitive and linguistic development. In particular the acquisition of new concepts, words and number representations.

Joshua B. Tenenbaum (Organizer) is Professor of Cognitive Science at MIT. Josh's lab sits at the intersection of cognitive science and machine learning, with a focus on hallmarks of human intelligence; in particular, the ability to learn rapidly and flexibly from limited data.

Noah D. Goodman is an Associate Professor of Psychology, Computer Science, and Linguistics at Stanford University. Noah's lab focuses on probabilistic programming, natural language semantics and pragmatics, intuitive theories and social cognition.

Laura Schulz is an Associate Professor of Cognitive Science at MIT. Laura's lab works on early childhood development of cognition, causal inference, discovery, and learning.

Steven T. Piantadosi is an Assistant Professor of Psychology at the University of Rochester. Steven's research uses formal computational methods and behavioral experiments to study how people learn language and create conceptual systems.

Marie Amalric is a Postdoctoral fellow advised by Jessica Cantlon at the University of Rochester. Marie studies the brain mechanisms involved in the learning of abstract mathematical concepts.

Anselm Rothe is a graduate student advised by Todd Gureckis and Brenden Lake at New York University. Anselm studies how humans and machines generate informative natural language questions.

Kevin Ellis is a graduate student advised by Josh Tenenbaum and Armando Solar-Lezama at MIT. Kevin's work examines how AI systems learn programs from data.

Ishita Dasgupta is a graduate student in Sam Gershman's Computational Cognitive Neuroscience lab. Ishita works on resource rational inference strategies in the domains of hypothesis generation, intuitive physics, and language.

Joshua Rule is a graduate student advised by Josh Tenenbaum at MIT. Josh uses computational models and behavioral experiments to study the development of conceptual systems.

Lucas E. Morales is a graduate student advised by Josh Tenenbaum at MIT. His work focuses on concept learning via program induction.

\section{Workshop structure}

We propose a full-day workshop consisting of 3 parts. The first two parts (morning and afternoon session) will be a series of 20-30 minute talks. The final part will be a panel discussion about the limits, open questions, and future of program induction in cognitive science.

The morning session will consist of the following talks:

\begin{tabular}{l|l}
\hline Presenter & Topic \\
\hline Bramley \& Schulz & Introductory remarks \\
Josh Tenenbaum & Cognition as program induction \\
Marie Amalric & A language of geometry \\
Lucas Morales & An architecture for program learning \\
Joshua Rule & Learning conceptual systems \\
Neil Bramley & Active data-driven concept learning \\
Fei Xu & Learning generalizations through play \\
\hline
\end{tabular}

The afternoon session will consist of the following talks:

\begin{tabular}{l|l}
\hline Presenter & Topic \\
\hline Steven Piantadosi & Language of thought \\
Eric Schulz & Learning list functions \\
Ishita Dasgupta & Compositionality in sentence embeddings \\
Kevin Ellis & Learning to learn programs \\
Anselm Rothe & Question asking as program induction \\
Laura Schulz & Discovery learning in childhood \\
Noah Goodman & Probabilistic program induction \\
\hline
\end{tabular}

The final 45 minutes will be a panel discussion led by the organizers with ample opportunity for audience participation.

\section{References}

Amalric, M., Wang, L., Pica, P., Figueira, S., Sigman, M., \& Dehaene, S. (2017). The language of geometry: Fast comprehension of geometrical primitives and rules in human adults and preschoolers. PLoS Computational Biology, 13(1), e1005273.

Bramley, N. R., Dayan, P., Griffiths, T. L., \& Lagnado, D. A. (2017). Formalizing Neurath's ship: Approximate algorithms for online causal learning. Psychological Review, 124(3), 301.

Bruner, J., Goodnow, J. J., \& Austin, G. (1956). A study of thinking.

Chater, N., \& Oaksford, M. (2013). Programs as causal models: Speculations on mental programs and mental representation. Cognitive Science, 37(6), 1171-1191.

Dechter, E., Malmaud, J., Adams, R. P., \& Tenenbaum, J. B. (2013). Bootstrap learning via modular concept discovery. In IJCAI, (pp. 1302-1309).

Ellis, K., Dechter, E., \& Tenenbaum, J. B. (2015). Dimensionality reduction via program induction. In 2015 AAAI Spring Symposium Series.

Goodman, N. D., Tenenbaum, J. B., Feldman, J., \& Griffiths, T. L. (2008). A rational analysis of rule-based concept learning. Cognitive Science, 32(1), 108-154.

Lake, B. M., Salakhutdinov, R., \& Tenenbaum, J. B. (2015). Humanlevel concept learning through probabilistic program induction. Science, 350(6266), 1332-1338.

Piantadosi, S. T., Tenenbaum, J. B., \& Goodman, N. D. (2016). The logical primitives of thought: Empirical foundations for compositional cognitive models. Psychological Review, 123(4), 392.

Romano, S., Salles, A., Amalric, M., Dehaene, S., Sigman, M., \& Figueria, S. (2017). Bayesian selection of grammar productions for the language of thought. bioRxiv, (p. 141358).

Rothe, A., Lake, B. M., \& Gureckis, T. (2017). Question asking as program generation. In Advances in Neural Information Processing Systems, (pp. 1046-1055).

Schulz, E., Tenenbaum, J. B., Duvenaud, D., Speekenbrink, M., \& Gershman, S. J. (2017). Compositional inductive biases in function learning. Cognitive Psychology, 99, $44-79$.

Sim, Z. L., \& Xu, F. (2017). Learning higher-order generalizations through free play: Evidence from 2-and 3-year-old children. Developmental Psychology, 53(4), 642. 